\title{
Photophysical properties of bichromophoric Fe(II) complexes bearing an aromatic electron acceptor
}

Antonio Francés-Monerris, ${ }^{1 *}$ Philippe C. Gros, ${ }^{2}$ Mariachiara Pastore,${ }^{1}$ Xavier Assfeld,,${ }^{1}$ and Antonio Monari ${ }^{1}$

1. Université de Lorraine, CNRS, LPCT, F54000 Nancy, France.

2. Université de Lorraine, CNRS, L2CM, F54000 Nancy, France.

Corresponding author: antonio.frances@univ-lorraine.fr

The replacement of heavy metals used by industry to produce optical devices would considerably reduce the environmental and economic cost of man-made technology. A possible strategy relies on the employment of lighter and more abundant metals like iron. The exploitability of the photophysics of Fe(II) complexes is, however, generally limited by their short excited-state lifetimes and poor emission properties. The present work studies the impact of appending an electron acceptor (anthracene) to an N-heterocyclic carbene (NHC) complex with the aim to trap the excited-state energy and, therefore, delay the excited-state decay of the considered iron compounds. Hence, the photophysical properties of six prototypes (built with different spacers between the NHC ligand and the anthracene moieties) have been studied by using time-dependent density functional theory and by determining the natural transition orbitals of the excited states. The computational results suggest that ethynyl bridges induce dual absorption properties, covering red and infrared wavelengths in addition to the violet-blue absorption of the metal-to-ligand charge transfer band, already reported for the parent compound. The nature of the lowest-lying triplet states indicates that, for all the considered prototypes, the excitation involves $\pi^{*}$ orbitals localized over anthracene, confirming its electron acceptor capabilities, and suggesting a possible equilibrium between different excited states that might lead to enhanced excited-state lifetimes and/or boosted luminescence properties.

\section{Introduction}

Exploitable photoactive devices based on organometallic complexes are often built using heavy and rare metals such as $\mathrm{Ru}(\mathrm{II}), \operatorname{Re}(\mathrm{I}), \mathrm{Os}(\mathrm{II})$ or Ir(III), mainly because of their optimal photophysical properties as, for example, high intersystem crossing yields, intense light emission or efficient electron transfer. The former properties may largely come from the inherent strong ligand field splitting of such metals that destabilizes the, in general, non-emissive metal-centered (MC) excited 
states. $[1,2]$ The resulting long-living metal-to-ligand charge transfer $\left({ }^{3} \mathrm{MLCT}\right)$ states are exploited in photoactive devices with applications in the fields of solar energy conversion,[3] molecular machines and switches, $[4,5]$ and luminescence.[6] The low abundance, and consequent high cost of precious metals, limits their massive use in technology, whereas their high toxicity threats the integrity of the biosphere. For these reasons, the scientific community is nowadays carrying out an active search for Earth-abundant metal complexes with exploitable photophysics able to match the needs of industry and to reduce at the same time the impact of human activity on the ecosystem. In this respect, ironbased complexes represents an attractive alternative to those based on $\mathrm{Ru}$, given the high abundance and relatively low toxicity of the former.[7]

In the last years, great efforts have been devoted to the comprehension of the excited-state dynamics of iron-based organometallic complexes. The use of time-resolved X-ray,[8-10] UV-vis and IR[11, 12] absorption and fluorescence up-conversion[13] spectroscopies, in combination with modern computational methods,[14-19] led to important breakthroughs in the understanding of the photoresponse, pointing to the crucial role of triplet and/or quintet MC states' population as the main pathways mediating the ultrafast non-radiative deactivation of the MLCT states. In addition, the comprehension of the inherent deactivation mechanisms has allowed the design of specific coordination spheres around iron able to extend the excited-state life-span of the complexes. Thus, the sub-picosecond lifetimes exhibited by the $\left[\mathrm{Fe}(\mathrm{bpy})_{3}\right]^{2+}$ prototype $[13,20]$ have been largely surpassed by the complexation with bidentate and tridentate N-heterocyclic carbene (NHC) ligands,[11, 21, 22] whereas a fine tuning of the excited-state lifetime, precisely relying on the fac/mer isomerism displayed by bidentate pyridylcarbene Fe(II) complexes, have been recently reported by our research group.[23, 24] In 2019, Kjær et al reported the coordination of Fe(III) with two monoanionic tris-carbene ligands to extend the lifetime of the corresponding doublet ${ }^{2} \mathrm{LMCT}$ state up to the nanosecond scale.[25]

In spite of the impressive advances in this direction, the functionalization of iron-based organometallic species with photochemical applications has received less attention so far, as compared to other metals. The development of versatile chromophores with tunable photophysical properties is of great interest in industry especially for its potential applicability in material science and engineering.[26-29] McClenaghan and coworkers have tuned the emission properties of systems based on copper[30, 31] and iridium[32, 33] by appending an organic chromophore (such as anthracene or pyrene) to one of the ligands coordinated with the metal center. As a result, the aromatic system serves as energy reservoir inducing an equilibrium between a number of excited-states, hence extending the excited-state lifetime by several orders of magnitude and enhancing the luminescence 
of the complexes, due to the radiative decay of the MLCT states. Regarding iron complexes, Brewer and coworkers[34] observed luminescence from a complex with 4-[N-(2-Anthryl)carbamoyl]-4'methyl-2,2'-bipyridine ligands, an analogue of the $\left[\mathrm{Fe}(\mathrm{bpy})_{3}\right]^{2+}$ prototype, $[13,20]$ demonstrating that the electron acceptor indeed captures the excitation energy even of ultrashort MLCT excited states, emitting the typical anthracene luminescence since the metal complex and the luminophore are not conjugated in this case.[34]

The present work explores the functionalization of $\mathrm{Fe}(\mathrm{II})-\mathrm{NHC}$ complexes by attaching electron acceptor groups to the tridentate NHC ligands aiming to trap the excitation energy and delay the excited-state decay, thus leading to $\mathrm{Fe}$ (II) complexes with different photophysical properties with respect to the traditional ones. We have chosen pyridyl-NHC-based ligands given their structural simplicity, good absorption of their Fe(II) complexes at 400-500 nm wavelengths and relatively longlived ${ }^{3}$ MLCT states up to $\sim 10$ ps.[35-37] The concept is tested using anthracene as an electron acceptor, linked to the NHC ligand by a number of spacers that allow or not the $\pi$-conjugation of the overall system,[38, 39] giving rise to the heteroleptic (1-6) series shown in Chart 1 (the parent compound $\mathbf{C O}$ is also shown).
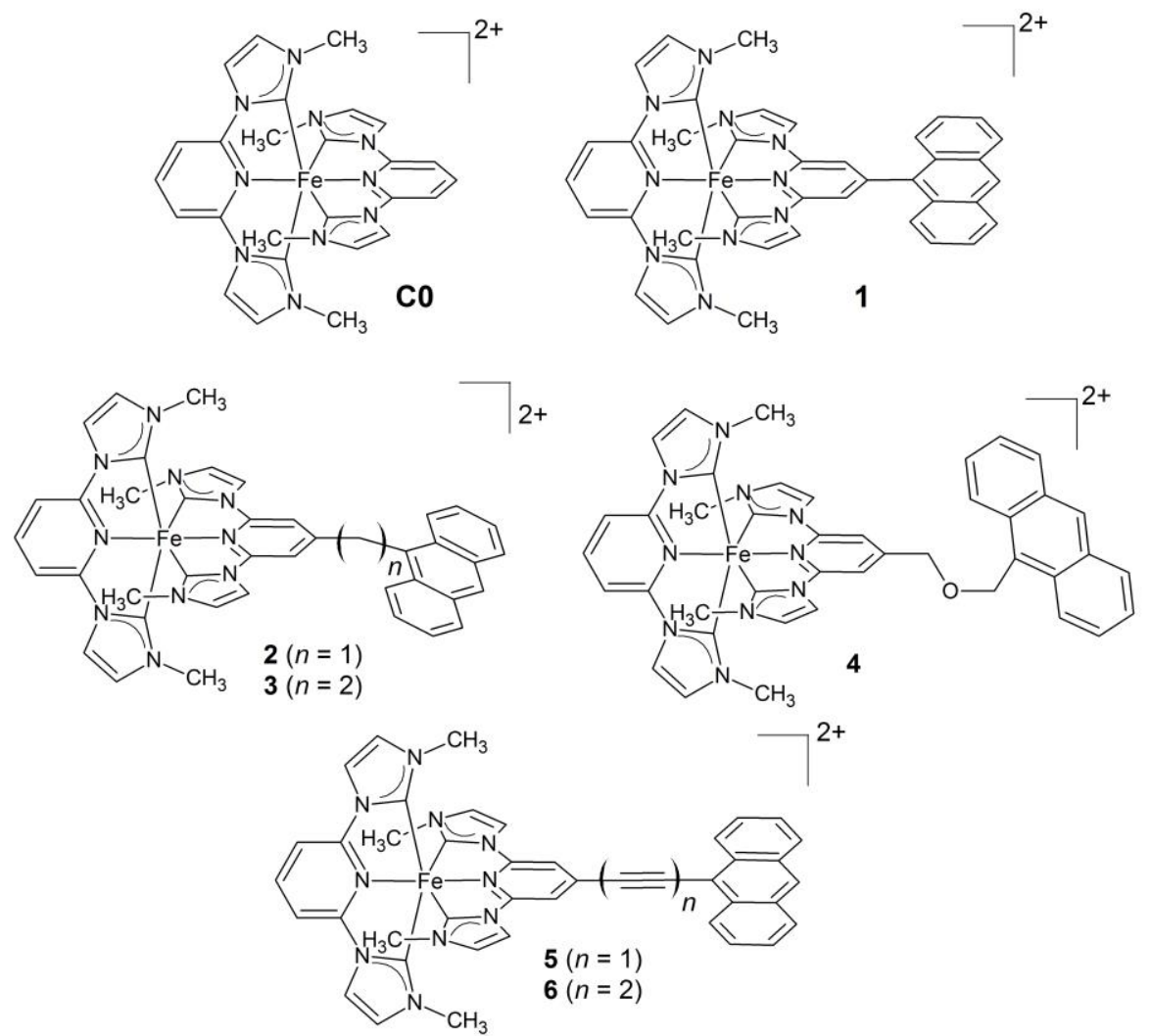

Chart 1. Chemical structures of the Fe(II) complexes studied in this work. 


\section{Computational methods}

All Fe(II) complexes have been optimized with the standard DFT/B3LYP method in conjunction with the $6-31 \mathrm{G}^{*}$ basis set. Harmonic vibrational frequencies have been computed at the same level of theory, and the optimized structures have been confirmed as minima by checking the absence of any normal mode with imaginary frequency. Unless otherwise stated, excited-state calculations have been carried out using time-dependent (TD)-DFT, in particular making use of the pure HCTH407 functional[40] (hereafter, TD-HCTH) and the larger 6-311G(d,p) basis set. The choice of this level of theory is based on the good description provided for similar Fe(II) compounds.[21-23, 35, 41] Other hybrid functionals, with different exact Hartree-Fock and DFT exchange ratio, such as B3LYP* $[15,42,43](15 \%)$ and B3LYP[44-46] (20\%), widely used to study inorganic complexes, have been tested and confirmed to provide a coherent description of the excited states (see Tables S1S3). To study the absorption properties, 40 singlet excited states have been computed as vertical transitions at the Franck-Condon geometry. To include the effects of thermal motion on the absorption spectra, the ground state potential energy surface has been sampled via a Wigner distribution, based on the harmonic vibrational frequencies and considering a temperature of 300 K.[47, 48] The sets of excitation energies and oscillator strengths obtained from 30 different structures have been subsequently convoluted using Gaussian functions of with a full-width at half-length of $0.3 \mathrm{eV}$.

The electronic density reorganization in the excited states have been analyzed in terms of natural transition orbitals (NTOs) [49, 50] through the Nancy_EX code.[50, 51] NTOs are obtained by singular value decomposition of the transition density matrix and constitute the best orbitals to represent an electronic transition. Contrary to the usual Kohn-Sham description, in the majority of cases the electronic density redistribution can be accounted by only one or two couples of occupied/virtual orbitals, allowing a much better definition of the excited state nature.[49]

The lowest-lying triplet states $\mathrm{T}_{1}$ have been optimized using the unrestricted DFT/HCTH ansatz (hereafter, $\mathrm{uHCTH}$ ) and the $6-31 \mathrm{G}^{*}$ basis set, whereas the final energies have been calculated with the TD-HCTH/6-311G(d,p) method on top of the uHCTH converged geometries. Negligible differences in the $\mathrm{T}_{1}$ description of $\mathbf{1}$ between the uDFT and the TD-DFT computational procedures have been found (see Table S4). The optimization carried out with the B3LYP functional led to a similar description of the $\mathrm{T}_{1}$ state.

All quantum-chemical calculations have been performed with the GAUSSIAN 09 (D.01 revision) software package.[52] Solvent effects (acetonitrile) have been included in all computations by means of the polarizable continuum model (PCM) with the default GAUSSIAN 09 parameters.[53] 


\section{Results and Discussion}

This section is organized as follows. First, we will provide a brief comparative analysis of the complexes' structures. Second, the UV/Vis absorption properties and the nature of the triplet states of the prototype 1 will be described in detail. Third, the impact of the linker between the NHC ligand and the electron acceptor on the absorption properties of 1-6 will be assessed. Finally, the lowestlying triplet states' nature and energy levels for the 1-6 series will be studied and discussed.

Structural features. The main structural difference between the tridentate complexes studied in this work is the relative alignment of the appended anthracene with respect to the NHC ligand, as displayed in Figure 1. This parameter strongly depends on the nature of the bridge. In complex $\mathbf{1}$, where there is no linker, the anthracene and ligand planes form a propeller angle of $72^{\circ}$ due to steric repulsions between the rings. For complexes $\mathbf{2 - 4}$, where the bridge is composed by carbon atoms with $\mathrm{sp}^{3}$ hybridation, the anthracene moiety has also a buckle angle (for instance, $\sim 109^{\circ}$ for $\mathbf{4}$, see Figure 1) arising from the pyramidalized atoms of the spacer. The exception is complex 2 , in which the ethyl bridge allows a propeller and buckle angles of $0^{\circ}$ even though the anthracene and the NHC systems are not conjugated. On the other hand, complexes 5 and $\mathbf{6}$ exhibit a planar arrangement typical of extended $\pi$-conjugated molecules.

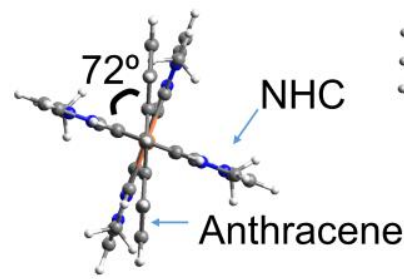

1

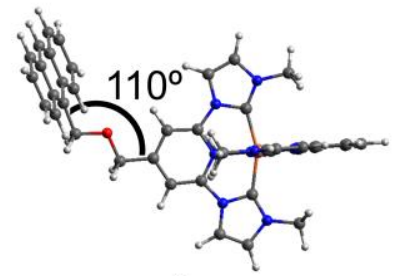

4

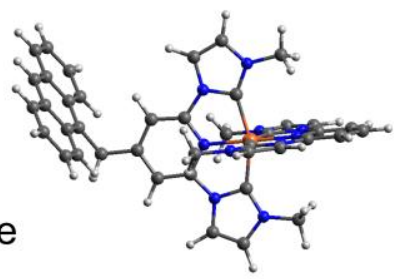

2

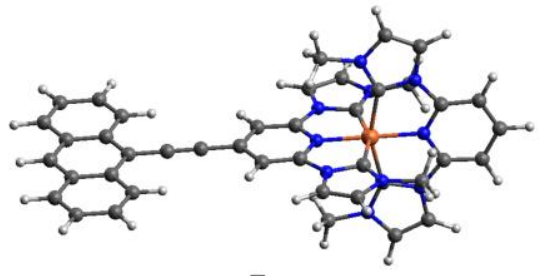

5

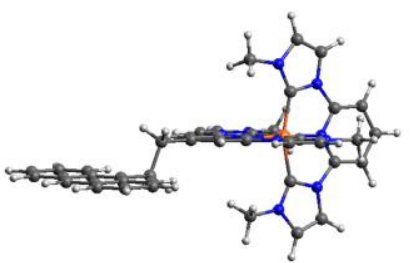

3

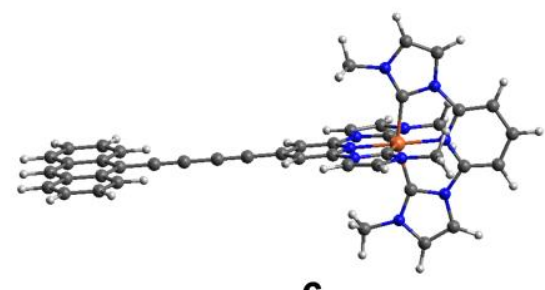

6

Figure 1. 3D geometries of complexes 1-6. The propeller and buckle angles are shown in complexes $\mathbf{1}$ and $\mathbf{4}$, respectively.

Linear optical absorption. The photophysical properties of the heteroleptic complex $\mathbf{1}$ will be evaluated in the first place due to the structural simplicity of this system, which lacks any linking bridge between the electron acceptor and the NHC ligand. The absorption spectrum is displayed in 
Figure 2. Two absorption bands are calculated in the visible range, an intense one centered at $437 \mathrm{~nm}$ and a shoulder centered at $c a .520 \mathrm{~nm}$. Analysis of the NTO topologies (see Figure 3) shows that the lowest-energy absorption is mainly due to excitation from anthracene to the pyridine ring of the NHC ligand, and to the metal in a lesser extent (hereafter, $\mathrm{A} \rightarrow \mathrm{NHC}$ transition). Some electronic density redistributions from the metal to the $\pi^{*}$ orbital of anthracene $(\mathrm{M} \rightarrow \mathrm{A})$ can also be identified, even though the oscillator strength of this transition for compound $\mathbf{1}$ is negligible. On the other hand, the much more intense absorption centered at $437 \mathrm{~nm}$ can be identified as the ${ }^{1}$ MLCT band typical of cyclometalated complexes and also described for the parent $\mathrm{Fe}(\mathrm{II})$ compound $\mathbf{C 0}$,[35] whose absorption spectrum is displayed in Figure 2 (grey line). It can be readily seen that the latter does not has the shoulder at $\sim 550 \mathrm{~nm}$ caused by excitations involving the anthracene unit, in coherence with the previous analysis of the NTO topologies for 1 reported in Figure 3. The differences between Co and $\mathbf{1}$ at $\sim 330 \mathrm{~nm}$, where $\mathbf{C O}$ exhibits a second small band, can be explained by the computational protocol adopted in this study. The 40 singlet excited states determined in this work do not cover the higher-energy MLCT states of $\mathbf{1}$ responsible for the absorption at this region, whereas they are computed for $\mathbf{C O}$ given the lower number of excited states in the latter complex. In summary, by considering the absorption spectra of $\mathbf{C O}$ and $\mathbf{1}$, and the fact that anthracene absorbs at much higher energies (ultraviolet region),[54] it can be safely concluded that the absorption spectrum of $\mathbf{1}$ is much closer to the one of the parent Fe(II) compound $\mathbf{C 0}[35]$ than to anthracene.

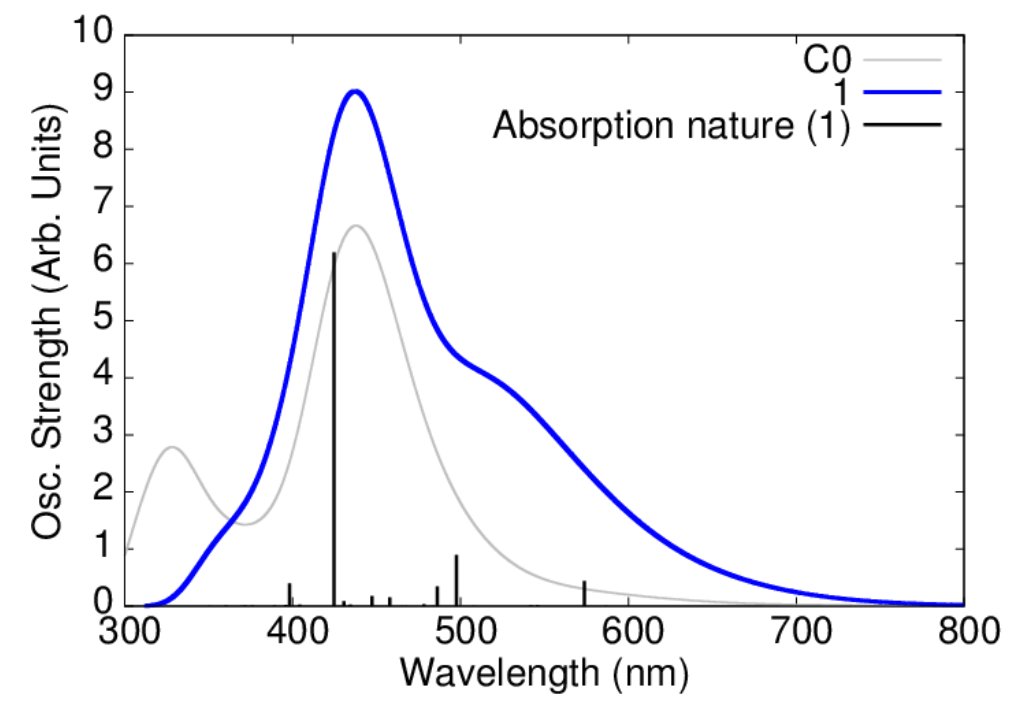

Figure 2. Absorption spectra of $\mathbf{C 0}$ (grey line) and $\mathbf{1}$ (blue line), and nature of the absorption bands of $\mathbf{1}$ (the black vertical lines correspond to the ground-state equilibrium geometry and the oscillator strength is not to scale). $\mathrm{M}=$ metal, $\mathrm{A}=$ acceptor (anthracene), $\mathrm{NHC}=\mathrm{N}$-heterocyclic carbene, MLCT $=$ metal-to-ligand charge transfer. 


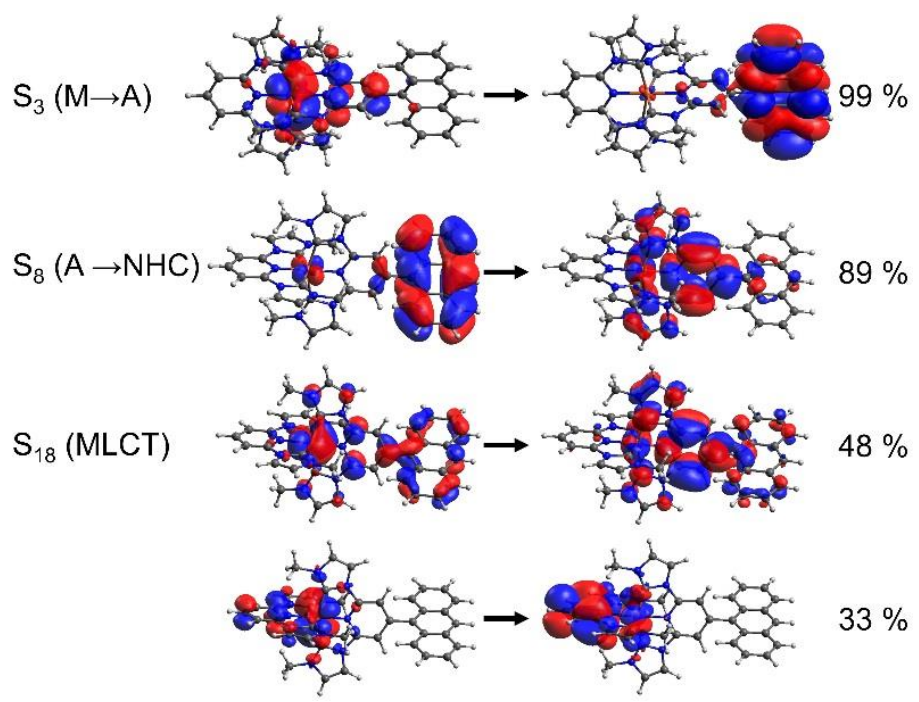

Figure 3. NTOs of the most relevant optical absorptions of $\mathbf{1}$. The percentage of each monoelectronic transition to the overall absorption is shown at the right.

The excitation energies at the Franck-Condon geometry of $\mathbf{1}$ were also calculated with other functionals containing different percentages of Hartree-Fock exchange, in particular 15\% (B3LYP*) and 20\% (B3LYP). All functionals predict two main absorption bands in the visible region, one of $\mathrm{A} \rightarrow \mathrm{NHC}$ nature at lower energies and the more intense MLCT band at higher energies, confirming the consistency of the results obtained with the pure HCTH functional. The main differences are ascribed to slight discrepancies in the ordering of the states and certain energy shifts, most likely due to the different Hartree-Fock exchange percentage included in the calculation. This conclusion is in agreement with previous studies on the determination of the triplet and singlet potential energy surfaces of bidentate Fe(II) complexes, where the performance of the HCTH and other hybrid functionals was assessed and the former showed the best agreement with the experimental measurements.[23]

Triplet energy levels of $\mathbf{1}$. For closed-shell transition metal complexes, time-resolved spectroscopy studies have repeatedly evidenced the occurrence of ultrafast intersystem crossings taking place after light absorption and leading to triplet population at the sub-picosecond regime.[8-11, 13, 21, 22, 24] Thus, after photoexcitation of $\mathbf{1}$ it is reasonable to expect a significant population of the triplet manifold that will eventually decay to the lowest-energy state, i.e. $\mathrm{T}_{1}$, via internal conversion. Hence the lowest lying triplet state has been studied via geometry optimization while its electronic structure has been analyzed considering the corresponding NTOs obtained on top of the converged equilibrium geometry. The orbitals are shown in Figure 4, and it can be readily seen that for $\mathrm{T}_{1}$ the excitation is localized over the anthracene molecule $\left(\pi_{\mathrm{A}} \rightarrow \pi^{*} \mathrm{~A}\right.$, hereafter $\left.\mathrm{A} \rightarrow \mathrm{A}\right)$, with only a minor participation 
of the metal atom. The fact that the lowest-energy triplet state is localized on anthracene already suggests that the presence of this electron acceptor will delay the overall excited-state decay of $\mathbf{1}$ acting as an energy reservoir, as reported for other metals.[30-33] The efficiency of this process will depend on the relative energy levels between the involved excited states, which will determine the equilibrium phenomena.

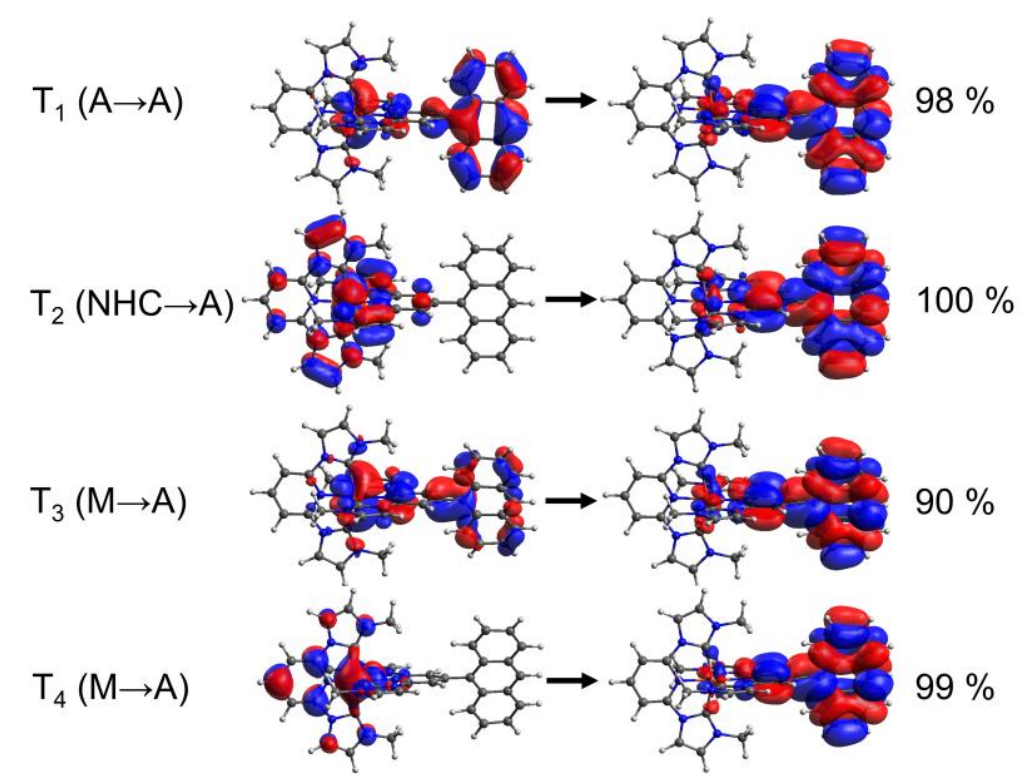

Figure 4. NTOs of the lowest-lying triplet states of $\mathbf{1}$ at the $\mathrm{T}_{1}$ equilibrium structure. The percentage of the dominant monoelectronic transitions is shown at the right.

The role of higher triplet states have been estimated by studying their nature and energy levels at the $\mathrm{T}_{1}$ equilibrium geometry (see Figure 4 and Table 1, respectively). Our results indicate that the states spanning the $\sim 1.5-2.0 \mathrm{eV}$ energy range from the ground state (see Table 1) are either localized entirely over anthracene $\left(\mathrm{T}_{1}\right)$, or involve charge-separation from the d metal orbitals to the $\pi^{*}$ orbital of the aromatic moiety (see Figure 4). This finding suggests anthracene as an efficient electron acceptor for the tridentate Fe-NHC complex, revealing its possible role as an energy reservoir in FeNHC complexes. It can be reasonably expected that this fact may significantly increase the excitedstate lifetimes and boost the emission properties of the cyclometalated systems. Indeed, with this triplet state being localized on anthracene, quenching by the metal-centered low-energy states should be largely reduced, and one may find a situation akin to the radiative properties of ${ }^{3}$ MLCT states in $\mathrm{Ru}$ or Ir. This hypothesis is also supported by the nature of the lowest-energy triplet states at the $\mathrm{S}_{0}$ equilibrium geometry (Figure S1), and of the lowest excited singlet states at the $\mathrm{T}_{1}$ minimum (see Figure S2), since all of them involve $\pi^{*}$ orbitals of anthracene. 
Table 1. Relative energies (eV) of the ground state and lowest-lying triplet states at the corresponding $\mathrm{S}_{0}$ and $\mathrm{T}_{1}$ equilibrium geometries of $\mathbf{1}$.

\begin{tabular}{lcccccc}
\hline & $\mathbf{S}_{\mathbf{0}}$ & $\mathbf{T}_{\mathbf{1}}$ & $\mathbf{T}_{\mathbf{2}}$ & $\mathbf{T}_{\mathbf{3}}$ & $\mathbf{T}_{\mathbf{4}}$ & $\mathbf{T}_{\mathbf{5}}$ \\
\hline $\mathbf{S}_{\mathbf{0}}$ min & 0.00 & 1.89 & 1.94 & 1.96 & 2.07 & 2.10 \\
$\mathbf{T}_{\mathbf{1}} \mathbf{m i n}$ & 0.17 & 1.56 & 1.80 & 1.95 & 1.98 & 2.29 \\
\hline
\end{tabular}

Absorption properties of the heteroleptic series (2-6). The possibility to introduce a linker between the anthracene and the NHC ligand and its impact on the absorption properties have been explored by means of systematic calculations performed in the 1-6 series of heteroleptic complexes (see Chart 1). Two types of bridges that break the aromatic conjugation have been used, namely alkyl ( 2 and $\mathbf{3})$ and ether (4) linkers. In contrast, the presence of one or two ethynyl groups (5 and $\mathbf{6}$, respectively) greatly extend the $\pi$ conjugation over the NHC and the anthracene moieties. Obviously, all of them increase the distance between anthracene and the iron atom. Results are compiled in Table 2. For $\mathbf{2}$, no significant absorptions below the MLCT transition at $2.92 \mathrm{eV}$ are found, whereas for $\mathbf{3}$, an absorption characterized by the charge transfer from the anthracene to the contiguous NHC ligand with significant oscillator strength is predicted at $2.46 \mathrm{eV}$, as it is the case for complex 1 (see Figure 3). The NTOs of the $\mathrm{A} \rightarrow \mathrm{NHC}$ excitations are displayed in Figure 5. Taking into account that the methyl and ethyl spacers do not imply aromatic conjugation between the NHC ligand and the anthracene systems, a possible explanation for the differences in the absorption properties observed between $\mathbf{1}, \mathbf{2}$, and $\mathbf{3}$, that should a priori display very similar optical properties, could be ascribed to the different relative arrangements between both NHC and anthracene chromophores. Thus, $\mathbf{1}$ has a perpendicular disposition between the two units, whereas in $\mathbf{2}$ the methyl linker, due to the $\mathrm{sp}^{3}$ hybridization, induces a distorted arrangement that precludes any coplanar arrangement between the NHC and the anthracene moieties, as can be seen in the geometries displayed in Figures 1 and 5. On the contrary, in complex $\mathbf{3}$, the larger flexibility added by the ethyl spacer allows for a coplanar arrangement between the NHC and the anthracene planes that maximizes the transition dipole moment of the absorption. Therefore, it becomes apparent that the different relative spatial orientations of the two chromophores strongly influences the oscillator strengths for the $\mathrm{A} \rightarrow \mathrm{NHC}$ transitions, perpendicular or coplanar arrangements seem to significantly increase absorption probability. Results for complex $\mathbf{4}$, with an ether spacer, are very similar to those of complex $\mathbf{2}$. This is indeed not surprising since the relative angle between the NHC and anthracene planes are similar in both compounds (see Figure 5). 
Table 2. Singlet absorption properties of the 1-6 complexes. For the sake of clarity, only states with oscillator strengths $(f) \geq 0.05$ are shown.

\begin{tabular}{cccccc}
\hline Complex & Adiabatic state & Nature & Energy $(\mathbf{e V})$ & $\boldsymbol{\lambda}(\mathbf{n m})$ & $\boldsymbol{f}$ \\
\hline $\mathbf{1}$ & $\mathrm{S}_{8}$ & $\mathrm{~A} \rightarrow \mathrm{NHC}$ & 2.49 & 498 & 0.0749 \\
& $\mathrm{~S}_{18}$ & MLCT & 2.92 & 425 & 0.5161 \\
$\mathbf{2}$ & $\mathrm{S}_{18}$ & MLCT & 2.92 & 424 & 0.5008 \\
$\mathbf{3}$ & $\mathrm{S}_{8}$ & $\mathrm{~A} \rightarrow \mathrm{NHC}$ & 2.46 & 504 & 0.0819 \\
& $\mathrm{~S}_{18}$ & $\mathrm{MLCT}$ & 2.94 & 421 & 0.4984 \\
$\mathbf{4}$ & $\mathrm{S}_{18}$ & $\mathrm{MLCT}$ & 2.94 & 422 & 0.4690 \\
$\mathbf{5}$ & $\mathrm{S}_{3}$ & $\mathrm{M} \rightarrow \mathrm{A}$ & 1.95 & 634 & 0.4642 \\
& $\mathrm{~S}_{5}$ & $\mathrm{~A} \rightarrow \mathrm{NHC}$ & 2.30 & 538 & 0.0457 \\
& $\mathrm{~S}_{6}$ & $\mathrm{~A} \rightarrow \mathrm{NHC}$ & 2.31 & 537 & 0.6560 \\
& $\mathrm{~S}_{16}$ & $\mathrm{MLCT}$ & 2.91 & 426 & 0.2050 \\
$\mathbf{6}$ & $\mathrm{S}_{3}$ & $\mathrm{M} \rightarrow \mathrm{A}$ & 1.88 & 659 & 0.6316 \\
& $\mathrm{~S}_{4}$ & $\mathrm{~A} \rightarrow \mathrm{NHC}$ & 2.12 & 585 & 0.8127 \\
& $\mathrm{~S}_{16}$ & MLCT & 2.81 & 442 & 0.4564 \\
\hline
\end{tabular}

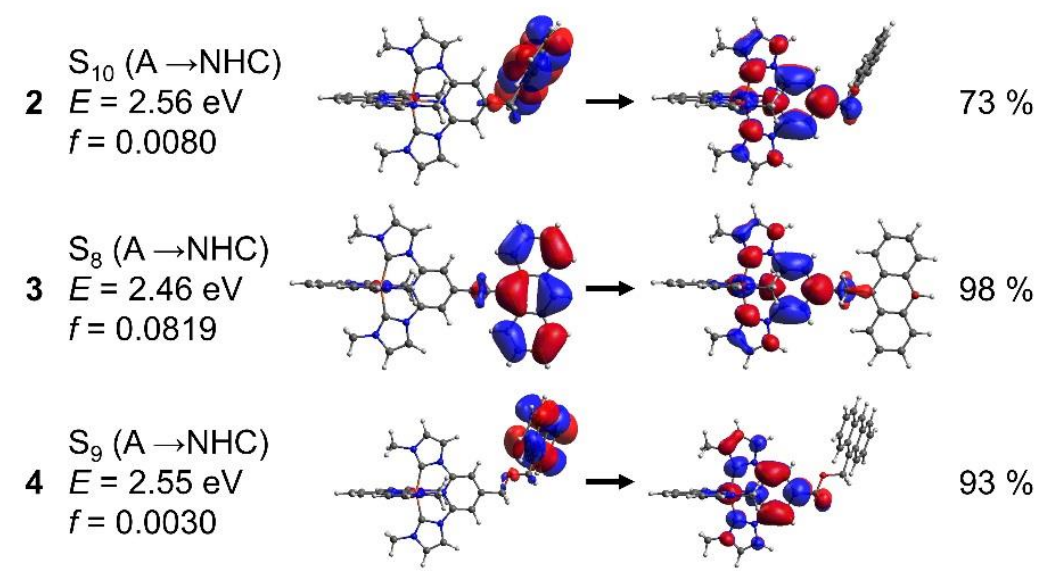

Figure 5. Excitation energies, oscillator strengths and NTOs of the $\mathrm{A} \rightarrow \mathrm{NHC}$ excitation of complexes $\mathbf{2 , 3}$ and 4 . The percentage of the dominant monoelectronic transitions is shown at the right.

The possible influence of the molecular vibrations in the absorption spectrum of $\mathbf{2}$ have been assessed by means of a Wigner sampling, as described in the computational details section. Results are shown in Figure 6. The computed spectra of $\mathbf{2}$ clearly confirms the lack of significant absorption at lower energies than the ${ }^{1}$ MLCT band. 


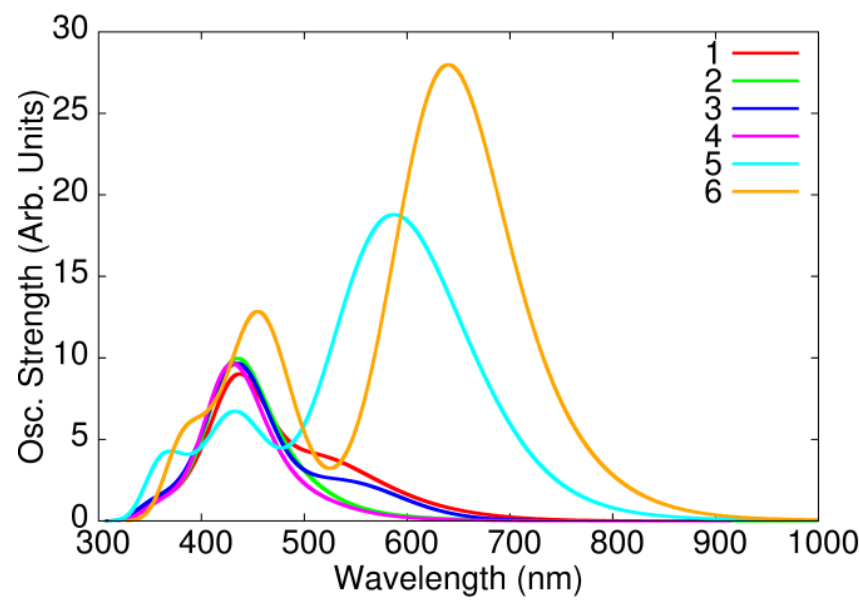

Figure 6. Absorption spectra of complexes 1-6.

In clear contrast to compounds $\mathbf{2 , 3}$, and $\mathbf{4}$, complexes 5 and $\mathbf{6}$ exhibit much more intense absorptions at low energies (see Table 2 and Figure 6) spanning the red and near infrared regions. These differences can clearly be ascribed to the presence of the one or two ethynyl linkers, which extend the $\pi$-conjugation of the anthracene system over the NHC ligand, dramatically decreasing the excitation energies of the larger aromatic super system. These results are in agreement with previous studies by Lapinte, Yam and collaborators[39, 55, 56] on the role of the 9,10-anthracenyl linker in electroactive systems based on non-octedral iron units, showing that the 9,10-anthracenyl group indeed acts as an efficient transmitter altering the original electronic properties of the complex.

As expected, and due to the extended conjugation, the ground state equilibrium geometry is characterized by a planar arrangement of the NHC ligand and anthracene (see Figures 1 and 7). A very bright state at low energies is identified as a metal-to-anthracene state (see Table 2), hereafter $\mathrm{M} \rightarrow \mathrm{A}$, which can be considered as a long-range MLCT state where the electron density hole is localized not only on the iron atom but also partially over the NHC ligand. Analogous states have also been characterized for $\mathbf{1}$ (see Figure 3), although much darker given the smaller extension of the $\pi$-system in this compound. On the other hand, the $\mathrm{A} \rightarrow \mathrm{NHC}$ absorptions reported for the nonconjugated complexes 1-4 are also present in the $\mathbf{5}$ and $\mathbf{6}$ compounds, and their intensity is also significantly enhanced due to the effective $\pi$-conjugation between the NHC ligand, the linkers and the anthracene systems. Therefore, it can be concluded that the addition of ethynyl linkers between the NHC and the anthracene fragments causes dual absorption properties in Fe(II) complexes, since in addition to the well-known MLCT band, an extra $\mathrm{M} \rightarrow \mathrm{A}$ intense absorption appears at lower energies. The synergy between the two absorption bands may lead to much more efficient light absorbers based on the abundant iron metal. 


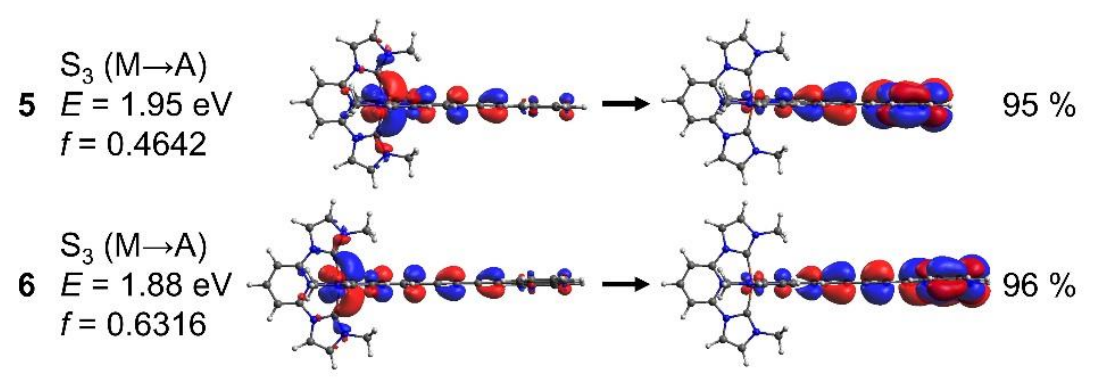

Figure 7. Excitation energies, oscillator strengths and NTOs of the $M \rightarrow A$ excitation of complexes 5 and 6. The percentage of the dominant monoelectronic transitions is shown at the right.

Analysis of the HOMO and LUMO energies and topologies, shown in Figure S3, reveals that, for all complexes, the HOMOs are localized mainly over the metal and that the LUMOs are centered in anthracene except for the parent $\mathbf{C 0}$, due to obvious reasons. The HOMO levels and topologies are not significantly influenced by the anthracene moiety and the different bridges, indicating no HOMO inversion in the 1-6 family. However, the different bridges do have a significant impact on the LUMO energies. In particular, the presence of the alkynyl group(s) clearly decrease the LUMO energy, coherently with the red-shift in the absorption bands observed for 5 and $\mathbf{6}$ computed with the TDDFT method in Figure 6.

Triplet energy levels of the heteroleptic series (2-6). As previously commented for $\mathbf{1}$, it is expected that the presence of an electron acceptor in complexes 2-6 will increase the excited-state lifetime and will favor luminescent pathways not observed in the parent Fe-NHC compounds.[21] The NTOs of the lowest-lying $T_{1}$ states at their corresponding equilibrium geometries for the complexes $\mathbf{2 - 6}$ are shown in Figure 8 . It can be readily seen that the spin density is mainly localized over the anthracene system $\mathrm{A} \rightarrow \mathrm{A}$, only in species $\mathbf{5}$ and $\mathbf{6}$ the unpaired electron distribution also involves, to some extent, the alkyne spacers and the iron atom even though their participation is of minor relevance. It shall be noted that the charge-transfer states of $\mathbf{6}$ have a pronounced long-range character and, consequently, they may be underestimated by pure GGA functionals like HCTH.[57-60] In order to ensure the nature of the lowest-lying triplet states, the $T_{1}$ state of $\mathbf{6}$ has been also computed with the long-range corrected hybrid CAM-B3LYP functional.[61] The NTOs are displayed in Figure S4 and confirm the $\mathrm{A} \rightarrow \mathrm{A}$ nature of the $\mathrm{T}_{1}$ state, showing a negligible participation of the metal. The next triplet state, i.e. $\mathrm{T}_{2}$, lies $\sim 1 \mathrm{eV}$ above $\mathrm{T}_{1}$, and the electronic density reorganization takes place mainly over the NHC 
moiety, whereas the lowest-energy ${ }^{3} \mathrm{MC}$ state is computed $1.15 \mathrm{eV}$ above $\mathrm{T}_{1}$ (relative energy of 2.89 $\mathrm{eV}$, see Figure S4).

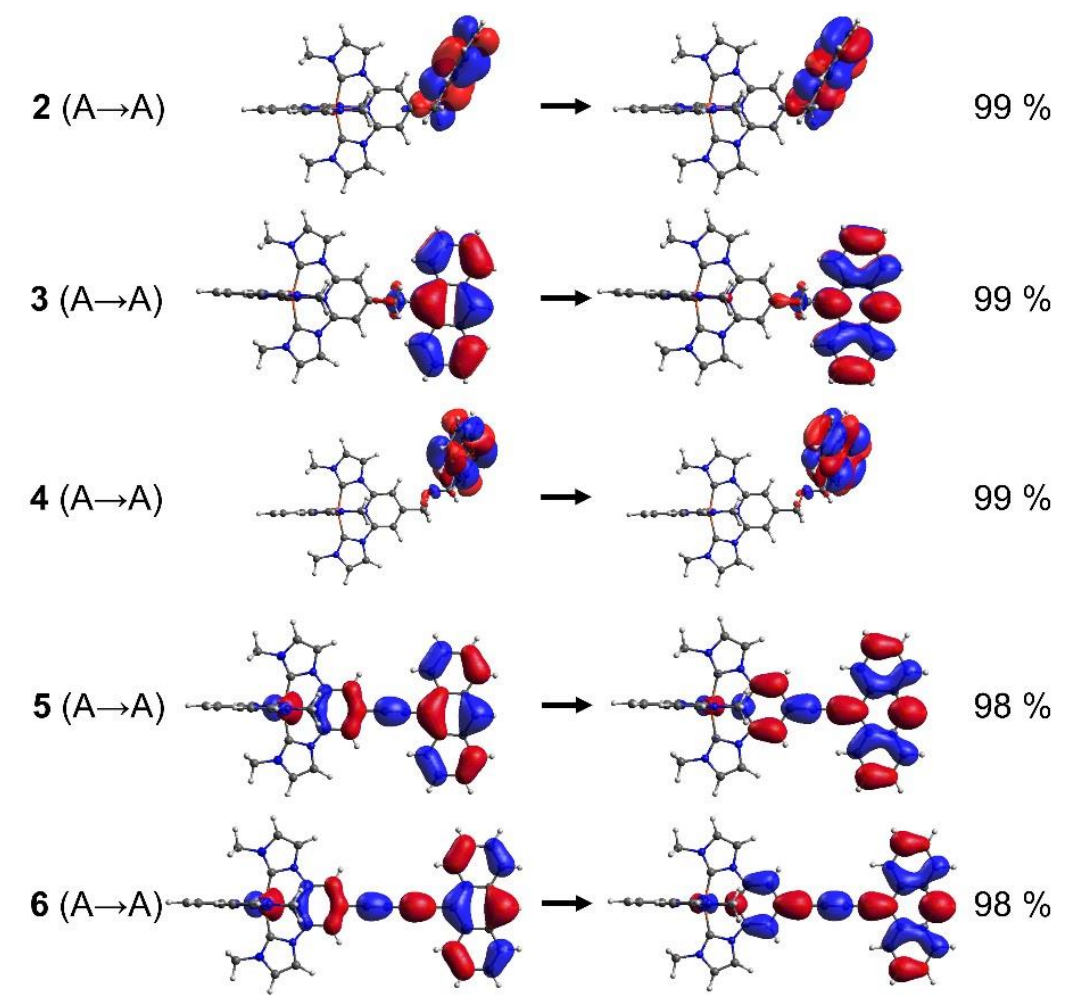

Figure 8. NTOs of the lowest-lying triplet states of 2-6 at their corresponding $\mathrm{T}_{1}$ equilibrium structure. The percentage of the dominant monoelectronic transitions is shown at the right.

The triplet energies for the 1-6 series are summarized in Table 3. The triplet relaxation does not induce significant energy changes with respect to the $T_{1}$ energy at the Franck-Condon geometry, indicating relatively small structural variations between the ground state and the triplet equilibrium structures. Interestingly, the triplet $\mathrm{A} \rightarrow \mathrm{A}$ energy levels of 5 and $\mathbf{6}$ lie below the ${ }^{3} \mathrm{MC}$ state minimum of the parent Fe-NHC compound determined at $1.29 \mathrm{eV}$ by Duchanois et al,[35] suggesting a much more efficient trapping of the excited state in these prototypes as compared to the 1-4 models, whose triplet $\mathrm{A} \rightarrow \mathrm{A}$ states lie slightly above this energy value. Finally, the vertical singlet-triplet gaps follow the same trend. Compounds 1-4 have similar $\mathrm{A} \rightarrow \mathrm{A}$ vertical energies $(1.39-1.47 \mathrm{eV})$, whereas 5 and $\mathbf{6}$ have a lower gap $(1.06-1.11 \mathrm{eV})$ due to the lower energy of the triplet states caused by the extended $\pi$ conjugation. 
Table 3. Relative energies and $S_{0}-T_{1}$ energy gaps and wavelength of the lowest-lying triplet states ${ }^{3}(\mathrm{~A} \rightarrow \mathrm{A})$ at their corresponding equilibrium geometries.

\begin{tabular}{ccc}
\hline Complex & $\begin{array}{c}\text { Relative energy } \\
(\mathbf{e V})\end{array}$ & $\begin{array}{c}\mathbf{S}_{\mathbf{0}}-\mathbf{T}_{\mathbf{1}} \text { energy gap } \\
(\mathbf{e V})\end{array}$ \\
\hline $\mathbf{1}$ & 1.56 & 1.39 \\
$\mathbf{2}$ & 1.65 & 1.47 \\
$\mathbf{3}$ & 1.63 & 1.46 \\
$\mathbf{4}$ & 1.64 & 1.45 \\
$\mathbf{5}$ & 1.21 & 1.11 \\
$\mathbf{6}$ & 1.14 & 1.06 \\
\hline
\end{tabular}

\section{Conclusions}

The photophysics of a series of octahedral Fe(II)-NHC complexes 1-6 bearing an anthracene molecule as electron acceptor has been studied by using DFT and TD-DFT methods and computing the natural transition orbitals of the excited states. Three types of linkers of alkyl, ether, and alkynyl nature have been considered to build the prototypes. Results reveal dual absorption properties in the visible range. One absorption band corresponds to the typical MLCT band previously reported for the parent FeNHC complexes[35] whereas an additional band involving the anthracene moiety is predicted at lower energies. The latter absorption is strongly influenced by the nature of the linker, since alkynyl spacers allow a large $\pi$-delocalization over the NHC ligands and anthracene extremely enhancing the absorption intensity of the band. On the other hand, a methyl or ether spacer strongly decreases the absorption capacities of this excitation.

The present work also reveals the nature of the lowest-lying triplet states, showing that the electron density rearrangement takes place mostly over the anthracene system. The triplet energy levels for the 1-6 models, especially those of compounds 5 and $\mathbf{6}$ which lie below the ${ }^{3} \mathrm{MC}$ state of the parent compound, suggest that the electron acceptor group may act as an energy reservoir delaying the excited-state decay of the complexes, leading thus to possible extended excited-state lifetimes and enhanced luminescence. In addition, it is crucial to underline how both absorption and emission in the infrared region are possible when extending the conjugation pathways, pointing to the possible use of such devices as efficient near-infrared probes. This study thus paves the way to the design of new photoactive compounds based in abundant metals, and shows that the strategy of appending electron acceptors to transition metal complexes, successfully applied to compounds based on metals like copper and iridium, represents a promising approach when is applied to Fe(II) systems. 


\section{Acknowledgements}

The authors thank Prof. Stefan Haacke (Université de Strasbourg) for his valuable suggestions and fruitful scientific discussions. This work has been funded by the French Agence Nationale de la Reserche (ANR) under the PhotIron project. A. F.-M. acknowledges the French Région Grand Est government and the ANR for post-doctoral contracts. All calculations have been performed using the local computing resources of the LPCT laboratory.

\section{References}

1. Wenger OS (2018) Photoactive Complexes with Earth-Abundant Metals. J Am Chem Soc 140:13522-13533. https://doi.org/10.1021/jacs.8b08822

2. Barbieri A, Accorsi G, Armaroli N (2008) Luminescent complexes beyond the platinum group: the d10 avenue. Chem Commun 2185-2193. https://doi.org/10.1039/B716650H

3. O'Regan B, Grätzel M (1991) A low-cost, high-efficiency solar cell based on dye-sensitized colloidal TiO2 films. Nature 353:737-740. https://doi.org/10.1038/353737a0

4. Browne WR, Feringa BL (2006) Making molecular machines work. Nat Nanotechnol 1:2535. https://doi.org/10.1038/nnano.2006.45

5. Colasson B, Credi A, Ragazzon G (2016) Light-driven molecular machines based on ruthenium(II) polypyridine complexes: Strategies and recent advances. Coord Chem Rev 325:125-134. https://doi.org/10.1016/j.ccr.2016.02.012

6. Kalinowski J, Fattori V, Cocchi M, Williams JAG (2011) Light-emitting devices based on organometallic platinum complexes as emitters. Coord Chem Rev 255:2401-2425. https://doi.org/10.1016/j.ccr.2011.01.049

7. Wenger OS (2019) Is Iron the New Ruthenium? Chem - A Eur J

0:doi:10.1002/chem.201806148. https://doi.org/10.1002/chem.201806148

8. Bressler C, Milne C, Pham V-T, et al (2009) Femtosecond XANES Study of the LightInduced Spin Crossover Dynamics in an Iron(II) Complex. Science (80- ) 323:489-492

9. Zhang W, Alonso-Mori R, Bergmann U, et al (2014) Tracking excited-state charge and spin dynamics in iron coordination complexes. Nature 509:345 
10. Kjaer KS, Kunnus K, Harlang TCB, et al (2018) Solvent control of charge transfer excited state relaxation pathways in [Fe(2,2'-bipyridine)(CN)4]2-. Phys Chem Chem Phys 20:42384249. https://doi.org/10.1039/C7CP07838B

11. Chábera P, Kjaer KS, Prakash O, et al (2018) FeII Hexa N-Heterocyclic Carbene Complex with a 528 ps Metal-to-Ligand Charge-Transfer Excited-State Lifetime. J Phys Chem Lett 459-463. https://doi.org/10.1021/acs.jpclett.7b02962

12. Monat JE, McCusker JK (2000) Femtosecond Excited-State Dynamics of an Iron(II) Polypyridyl Solar Cell Sensitizer Model. J Am Chem Soc 122:4092-4097. https://doi.org/10.1021/ja992436o

13. Gawelda W, Cannizzo A, Pham V-T, et al (2007) Ultrafast Nonadiabatic Dynamics of [Fe II (bpy) $\left.{ }_{3}\right]^{2+}$ in Solution. J Am Chem Soc 129:8199-8206. https://doi.org/10.1021/ja070454x

14. Ashley DC, Jakubikova E (2017) Ironing out the photochemical and spin-crossover behavior of Fe(II) coordination compounds with computational chemistry. Coord Chem Rev 337:97111. https://doi.org/10.1016/j.ccr.2017.02.005

15. Fredin LA, Pápai M, Rozsályi E, et al (2014) Exceptional excited-state lifetime of an iron(II)- N -heterocyclic carbene complex explained. J Phys Chem Lett 5:2066-2071. https://doi.org/10.1021/jz500829w

16. Sousa C, De Graaf C, Rudavskyi A, et al (2013) Ultrafast deactivation mechanism of the excited singlet in the light-induced spin crossover of [Fe(2,2-bipyridine)3]2+. Chem - A Eur J 19:17541-17551. https://doi.org/10.1002/chem.201302992

17. Graaf C De, Sousa C (2011) On the role of the metal-to-ligand charge transfer states in the light-induced spin crossover in FeII (bpy)3. Int J Quantum Chem 111:3385-3393. https://doi.org/10.1002/qua.22991

18. Pápai M, Vankó G, de Graaf C, Rozgonyi T (2013) Theoretical Investigation of the Electronic Structure of Fe(II) Complexes at Spin-State Transitions. J Chem Theory Comput 9:509-519. https://doi.org/10.1021/ct300932n

19. Nance J, Bowman DN, Mukherjee S, et al (2015) Insights into the Spin-State Transitions in [Fe(tpy)2]2+: Importance of the Terpyridine Rocking Motion. Inorg Chem 54:11259-11268. https://doi.org/10.1021/acs.inorgchem.5b01747

20. Lees AJ, Long C (2010) Photophysics of Organometallics. Top Organomet Chem 29:37-71. https://doi.org/10.1007/978-3-642-04729-9 
21. Duchanois T, Liu L, Pastore M, et al (2018) NHC-Based Iron Sensitizers for DSSCs. Inorganics 6:63. https://doi.org/10.3390/inorganics6020063

22. Liu L, Duchanois T, Etienne T, et al (2016) A new record excited state ${ }^{3}$ MLCT lifetime for metalorganic iron( II ) complexes. Phys Chem Chem Phys 18:12550-12556.

https://doi.org/10.1039/C6CP01418F

23. Francés-Monerris A, Magra K, Darari M, et al (2018) Synthesis and Computational Study of a Pyridylcarbene Fe(II) Complex: Unexpected Effects of fac/mer Isomerism in Metal-toLigand Triplet Potential Energy Surfaces. Inorg Chem 57:10431-10441

24. Magra K, Domenichini E, Francés-Monerris A, et al (2019) Impact of the fac/mer Isomerism on the Excited-State Dynamics of Pyridyl-carbene Fe(II) Complexes. Inorg Chem 58:50695081. https://doi.org/10.1021/acs.inorgchem.9b00138

25. Kjær KS, Kaul N, Prakash O, et al (2019) Luminescence and reactivity of a charge-transfer excited iron complex with nanosecond lifetime. Science (80- ) 363:249 LP - 253. https://doi.org/10.1126/science.aau7160

26. Zhang X, Chi Z, Zhang Y, et al (2013) Recent advances in mechanochromic luminescent metal complexes. J Mater Chem C 1:3376-3390. https://doi.org/10.1039/C3TC30316K

27. Dong Y, Zhang J, Tan X, et al (2013) Multi-stimuli responsive fluorescence switching: the reversible piezochromism and protonation effect of a divinylanthracene derivative. J Mater Chem C 1:7554-7559. https://doi.org/10.1039/C3TC31553C

28. Londesborough MGS, Dolanský J, Cerdán L, et al (2017) Thermochromic Fluorescence from B18H20(NC5H5)2: An Inorganic-Organic Composite Luminescent Compound with an Unusual Molecular Geometry. Adv Opt Mater 5:1600694. https://doi.org/10.1002/adom.201600694

29. Shi C, Zhu Y, Zhu G, et al (2018) Phototunable full-color emission of dynamic luminescent materials. J Mater Chem C 6:9552-9560. https://doi.org/10.1039/C8TC02955E

30. Leydet Y, Bassani DM, Jonusauskas G, McClenaghan ND (2007) Equilibration between Three Different Excited States in a Bichromophoric Copper(I) Polypyridine Complex. J Am Chem Soc 129:8688-8689. https://doi.org/10.1021/ja072335n

31. Armaroli N (2008) Electronic Excited-State Engineering. ChemPhysChem 9:371-373. https://doi.org/10.1002/cphc.200700794

32. Denisov SA, Cudré Y, Verwilst P, et al (2014) Direct Observation of Reversible Electronic 
Energy Transfer Involving an Iridium Center. Inorg Chem 53:2677-2682.

https://doi.org/10.1021/ic4030712

33. Yarnell JE, McCusker CE, Leeds AJ, et al (2016) Exposing the Excited-State Equilibrium in an IrIII Bichromophore: A Combined Time Resolved Spectroscopy and Computational Study. Eur J Inorg Chem 2016:1808-1818. https://doi.org/10.1002/ejic.201600194

34. Zigler DF, Elvington MC, Heinecke J, Brewer KJ (2006) Luminescently Tagged 2,2`Bipyridine Complex of FeII: Synthesis and Photophysical Studies of 4-[N-(2Anthryl)carbamoyl]-4‘-methyl-2,2‘-bipyridine. Inorg Chem 45:6565-6567. https://doi.org/10.1021/ic060207e

35. Duchanois T, Etienne T, Cebrián C, et al (2015) An Iron-Based Photosensitizer with Extended Excited-State Lifetime: Photophysical and Photovoltaic Properties: An Iron-Based Photosensitizer with Extended Excited-State Lifetime. Eur J Inorg Chem 2015:2469-2477. https://doi.org/10.1002/ejic.201500142

36. Pastore M, Duchanois T, Liu L, et al (2016) Interfacial charge separation and photovoltaic efficiency in Fe(II)-carbene sensitized solar cells. Phys Chem Chem Phys 18:28069-28081. https://doi.org/10.1039/c6cp05535d

37. Liu Y, Harlang T, Canton SE, et al (2013) Towards longer-lived metal-to-ligand charge transfer states of iron(ii) complexes: an N-heterocyclic carbene approach. Chem Commun 49:6412. https://doi.org/10.1039/c3cc43833c

38. Hunter CA, Lawson KR, Perkins J, Urch CJ (2001) Aromatic interactions. J Chem Soc \{,\} Perkin Trans 2 651-669. https://doi.org/10.1039/B008495F

39. Halet J-F, Lapinte C (2013) Charge delocalization vs localization in carbon-rich iron mixedvalence complexes: A subtle interplay between the carbon spacer and the (dppe) $\mathrm{Cp} * \mathrm{Fe}$ organometallic electrophore. Coord Chem Rev 257:1584-1613. https://doi.org/https://doi.org/10.1016/j.ccr.2012.09.007

40. Boese AD, Handy NC (2001) A new parametrization of exchange-correlation generalized gradient approximation functionals. J Chem Phys 114:5497-5503. https://doi.org/10.1063/1.1347371

41. Duchanois T, Etienne T, Beley M, et al (2014) Heteroleptic Pyridyl-Carbene Iron Complexes with Tuneable Electronic Properties. Eur J Inorg Chem 2014:3747-3753. https://doi.org/10.1002/ejic.201402356 
42. Kepp KP (2016) Theoretical Study of Spin Crossover in 30 Iron Complexes. Inorg Chem 55:2717-2727. https://doi.org/10.1021/acs.inorgchem.5b02371

43. Dixon IM, Alary F, Boggio-Pasqua M, Heully J-L (2015) Reversing the relative 3MLCT$3 \mathrm{MC}$ order in $\mathrm{Fe}$ (II) complexes using cyclometallating ligands: a computational study aiming at luminescent Fe(II) complexes. Dalt Trans 44:13498-13503. https://doi.org/10.1039/C5DT01214G

44. Mukherjee S, Torres DE, Jakubikova E (2017) HOMO inversion as a strategy for improving the light-absorption properties of Fe(II) chromophores. Chem Sci 8:8115-8126. https://doi.org/10.1039/C7SC02926H

45. Bowman DN, Bondarev A, Mukherjee S, Jakubikova E (2015) Tuning the Electronic Structure of Fe(II) Polypyridines via Donor Atom and Ligand Scaffold Modifications: A Computational Study. Inorg Chem 54:8786-8793. https://doi.org/10.1021/acs.inorgchem.5b01409

46. Bedford RB, Brenner PB, Carter E, et al (2014) Iron Phosphine Catalyzed Cross-Coupling of Tetraorganoborates and Related Group 13 Nucleophiles with Alkyl Halides. Organometallics 33:5767-5780. https://doi.org/10.1021/om500518r

47. Dahl JP, Springborg M (1988) The Morse oscillator in position space, momentum space, and phase space. J Chem Phys 88:4535-4547. https://doi.org/10.1063/1.453761

48. Barbatti M, Sen K (2016) Effects of different initial condition samplings on photodynamics and spectrum of pyrrole. Int J Quantum Chem 116:762-771.

https://doi.org/10.1002/qua.25049

49. Martin RL (2003) Natural transition orbitals. J Chem Phys 118:4775-4777. https://doi.org/10.1063/1.1558471

50. Etienne T, Assfeld X, Monari A (2014) Toward a quantitative assessment of electronic transitions" charge-transfer character. J Chem Theory Comput 10:3896-3905. https://doi.org/10.1021/ct5003994

51. Etienne T, Assfeld X, Monari A (2014) New Insight into the Topology of Excited States through Detachment/Attachment Density Matrices-Based Centroids of Charge. J Chem Theory Comput 10:3906-3914. https://doi.org/10.1021/ct500400s

52. Frisch MJ, Trucks GW, Schlegel HB, et al (2010) Gaussian09. Revis D01 Gaussian Inc., Wallingford CT 
53. Tomasi J, Mennucci B, Cammi R (2005) Quantum mechanical continuum solvation models. Chem Rev 105:2999-3093. https://doi.org/10.1021/cr9904009

54. Jones RN (1947) The Ultraviolet Absorption Spectra of Anthracene Derivatives. Chem Rev 41:353-371. https://doi.org/10.1021/cr60129a013

55. de Montigny F, Argouarch G, Roisnel T, et al (2008) Syntheses, Structures, and Properties of Some Piano-Stool Iron Acetylides Bearing a Functional Anthracenyl Group.

Organometallics 27:1912-1923. https://doi.org/10.1021/om701278e

56. de Montigny F, Argouarch G, Costuas K, et al (2005) Electron Transfer and Electron Exchange between $[\mathrm{Cp} *(\mathrm{dppe}) \mathrm{Fe}] \mathrm{n}+(\mathrm{n}=0,1)$ Building Blocks Mediated by the 9,10Bis(ethynyl)anthracene Bridge. Organometallics 24:4558-4572. https://doi.org/10.1021/om050403d

57. Dreuw A, Weisman JL, Head-Gordon M (2003) Long-range charge-transfer excited states in time-dependent density functional theory require non-local exchange. J Chem Phys 119:2943-2946. https://doi.org/10.1063/1.1590951

58. Tozer DJ (2003) Relationship between long-range charge-transfer excitation energy error and integer discontinuity in Kohn-Sham theory. J Chem Phys 119:12697-12699. https://doi.org/10.1063/1.1633756

59. Pastore M, Mosconi E, De Angelis F, Grätzel M (2010) A Computational Investigation of Organic Dyes for Dye-Sensitized Solar Cells: Benchmark, Strategies, and Open Issues. J Phys Chem C 114:7205-7212. https://doi.org/10.1021/jp100713r

60. Ronca E, Angeli C, Belpassi L, et al (2014) Density Relaxation in Time-Dependent Density Functional Theory: Combining Relaxed Density Natural Orbitals and Multireference Perturbation Theories for an Improved Description of Excited States. J Chem Theory Comput 10:4014-4024. https://doi.org/10.1021/ct5004675

61. Yanai T, Tew DP, Handy NC (2004) A new hybrid exchange-correlation functional using the Coulomb-attenuating method (CAM-B3LYP). Chem Phys Lett 393:51-57. https://doi.org/https://doi.org/10.1016/j.cplett.2004.06.011 